\title{
Las Figuras Docentes de la Formación de Médicos Especialistas ante el Real Decreto 183/2008: El Jefe de Residentes y el Técnico Docente
}

\author{
José Saura Llamas ${ }^{a}$, José Galcerá Tomás ${ }^{b}$, Carmen Botella Martínez ${ }^{c}$ y \\ Miembros del Foro de Jefes de Estudios de la Región de Murcia ${ }^{d}$
}

a Técnico de Salud de la Unidad Docente de Medicina Familiar y Comunitaria de Murcia. España.

b Jefe de Estudios de Formación Especializada. Hospital Universitario "Virgen de la Arrixaca", Murcia. España.

c Técnica de Apoyo Docente. Hospital Universitario "Virgen de la Arrixaca”, Murcia. España.

d Fernando Navarro Mateu, Iñigo Anza Aguirrezabala, Andrés Conesa Hernández, Faustino Herrero Huerta,

Jacinto Fernández Pardo, Julio Fontcuberta Martínez, Antonio Martínez Pastor, Magdalena Molina Oller y

Andrés Carrillo Alcaraz.

Correspondencia: José Saura Llamas, c/ Escultor Sánchez Lozano no 5, 30007-Murcia (España). Correo electrónico: j.saurall@gmail.com

Recibido el 29 de septiembre de 2010.

Aceptado para su publicación el 4 de noviembre de 2010 .

Del "Taller de actualización en la Normativa sobre las Figuras Docentes en la Formación Sanitaria Especializada" celebrado en Murcia en febrero de 2010.

\section{RESUMEN}

El Real Decreto 183/2008 sobre Formación Médica Especializada en Ciencias de la Salud ha supuesto un cambio sustancial de esta formación en nuestro país. Desde su publicación, las Unidades Docentes (UU DD) han comenzado un proceso de adaptación a este decreto, proceso que es complejo y que requiere mucho esfuerzo ante los múltiples e importantes cambios que introduce.

Este viaje presenta dificultades añadidas por el incumplimiento de las instituciones competentes de elaborar la normativa que desarrolle la mayoría de sus contenidos, y por lo tanto las UU DD carecen de las guías y orientaciones al respecto.

Como iniciativa de la Comisión de Docencia de Formación Especializada del Hospital Universitario Virgen de la Arrixaca, se presenta el resultado del trabajo en grupo de los asistentes (informadores clave) al taller de Actualización en la Normativa sobre las Figuras Docentes en la Formación Sanitaria Especializada celebrado en Murcia, febrero de 2010. En este caso se centra en las propuestas elaboradas sobre las figuras del Jefe de Residentes y el Técnico Docente, con la intención de aportar ayuda para los jefes de estudios y los docentes en esta adaptación.

Palabras clave. Educación, Especialización, Programas de Postgrado en Salud.

\section{ABSTRACT}

Educators in Specialised Medical Training in view of the Royal Decree 183/2008: The Head of Residents in Training and the Teacher

The Spanish Royal Decree 183/2008 on Specialised Medical Training in Health Sciences has meant a substantial change to this kind of training in Spain. Since its publication, the Teaching Units (TUs) have started a process of adaptation to this decree, a process that is complex and that requires much effort in view of the many significant changes it introduces.

The process is complicated even more because the competent institutions have failed to develop regulation on most of the Decree's contents, leaving the TUs with no guidelines.

As an initiative of the Education Committee on Specialised Training of the Hospital Universitario Virgen de la Arrixaca we present the results of the participant's teamwork (key informers) in a workshop on the Update of the Regulation on Educators in Specialised Health Training that took place in Murcia in February 2010. In this case we focus on the proposals developed for the Head of Residents in training and Teachers, with the aim of providing support to the heads of studies and teachers in this adaptation.

Key Words. Education, Specialization, Health Postgraduate Programs.

\section{INTRODUCCIÓN}

El sistema MIR de formación médica especializada se inició en nuestro país en los años 60 de una manera minoritaria, y se extendió dentro del Sistema Nacional de Salud tras varias experiencias piloto de éxito. El conjunto de la formación MIR adquirió carta de naturaleza con la promulgación del RD 127 de $1984^{1}$, en el que se estructuraba y regulaba esta formación, aunque de una manera muy general.

En la orden de $1995^{2}$ fueron reconocidos los diferentes aspectos y mencionadas por primera vez las diferentes figuras participantes en la formación especializada. 
Paradójicamente, y a pesar de la existencia de ésta y otra abundante normativa, la formación MIR era globalmente una formación poco regulada y escasamente normalizada, con una muy pobre definición del proceso formativo, con una ausencia muy llamativa de datos e información sobre el seguimiento y la evaluación del proceso formativo, y sobre todo con una falta de definición y descripción detallada de las funciones de las distintas figuras docentes.

Esta situación, añadida a la necesaria adaptación al Espacio Europeo de Formación Superior, ha hecho que recientemente hayan aparecido nuevas normas, entre las que destaca el RD 183 de $2008^{3}$, que han introducido cambios sustanciales en el proceso de Evaluación, el control de calidad de la formación, en las funciones de algunas de las figuras docentes, y especialmente en la ampliación de las competencias del papel del tutor ${ }^{3}$, descritas con mucho detalle, así como las funciones del Jefe de Estudios ${ }^{3}$, compartidas o no con la Comisión de Docencia correspondiente.

El artículo 13 del citado RD 183 de $2008^{3}$, titulado "otras figuras docentes", recoge la posibilidad de que las comunidades autónomas, según sus características y criterios organizativos propios, puedan crear otras figuras docentes con la finalidad de amparar colaboraciones significativas en la formación especializada, objetivos de investigación, desarrollo de módulos genéricos o específicos de los programas o cualesquiera otras actividades docentes de interés, aunque no menciona expresamente a algunas figuras que ya existen dentro del sistema MIR como son el Técnico de Salud Pública o Técnico de Apoyo Docente y el Jefe de Residentes.

Pero la actual normativa ha dejado muchos aspectos, con gran repercusión práctica en el desempeño de los roles docentes, a la publicación de nuevas normas por las diferentes comunidades autónomas (CCAA) de acuerdo con las disposiciones de la Ley de Ordenación de Profesiones Sanitarias ${ }^{4}$. Sin embargo, el plazo contemplado en el RD 183/2008 para el desarrollo de dicha normativa autonómica finalizó en febrero de $2009^{3}$.

Aunque este incumplimiento ha supuesto un freno a la posible implantación al desarrollo de la normativa y a la mejora del proceso formativo de los MIR, como aspecto positivo, ha permitido, disponer de un tiempo de reflexión para que los responsables de las CCAA puedan tener en cuenta la opinión de los principales actores de la formación especiali- zada con relación al desempeño de los roles de las distintas figuras docentes. Curiosamente, y a pesar de que estos colectivos de docentes (sobre todo los tutores) tienen una gran experiencia en el desempeño de estas labores, hasta ahora prácticamente no han sido consultados para elaborar estas normas.

\section{MATERIAL Y MÉTODOS}

Ante esta situación la Comisión de Docencia Especializada del Hospital Universitario "Virgen de la Arrixaca", a través de su Jefe de Estudios, y con la intención de recoger las opiniones de los docentes que vienen desempeñando dichas tareas formativas, puso en marcha una investigación cualitativa durante el mes de Febrero de 2010, bajo la forma de un Taller denominado de actualización de la normativa sobre las figuras docentes en la Formación Sanitaria Especializada.

Esta actividad ha constado de 3 sesiones de 5 horas, desarrolladas en tres días en horario de tarde, y en cada una de ellas se ha tratado una de las siguientes 3 figuras docentes: El Jefe de Residentes, El Técnico Docente y el Jefe de Estudios (y la Comisión de Docencia) . En ellas han participado como informadores clave: Jefes de Estudios de todas las Unidades Docentes de Formación Especializada, técnicos de salud y técnicos docentes, jefes de residentes, tutores y otros docentes, de la CA de la Región de Murcia y de otras CCAA del ámbito nacional. Con 43 inscritos y una participación media de 30 personas.

La actividad se desarrollaba en 2 partes: en una primera parte se realizaba una exposición por varios docentes expertos en cada una de las tres figuras docentes objeto del taller, de $1 \frac{1}{2}$ hora de duración, exponiéndose los orígenes, la situación actual y propuestas para su desarrollo de cada una de ellas.

En la segunda parte, de unas 3 horas de duración, se desarrollaba un trabajo en grupo, con un moderador que además recogía los resultados, bajo la doble forma de la tormenta de ideas y el grupo nominal, elaborándose al finalizar cada sesión un documento de consenso con las conclusiones y propuestas concretas de estos docentes sobre a la definición, funciones y otras características deseables para estas figuras docentes. Los resultados referidos al Jefe de Residentes y al Técnico Docente son los que se exponen en este artículo. 


\section{EL JEFE DE RESIDENTES}

\section{Orígenes y justificación}

A finales del siglo XVIII surge en el Johns Hopkins Hospital el embrión de esta figura ("Chief of Residents"), y es en EE.UU. y Canadá donde adquiere consistencia y se establece propiamente como un puesto de importancia y renombre. En España el hospital Ramón y Cajal ha sido pionero en este aspecto.

Actualmente en nuestro país se trata de una figura no legislada que corresponde a un facultativo especialista que acaba de finalizar su formación como residente y es contratado por el periodo de un año.

En los centros en los que existe esta figura se ha considerado una experiencia positiva la participación del Jefe de Residentes en la docencia, ya que contribuye a implicar al colectivo de residentes en la organización de la docencia y a una participación activa en la misma.

\section{Conclusiones y propuestas}

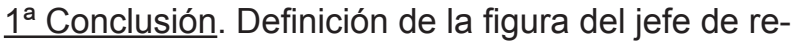
sidentes:

El Jefe de Residentes se sitúa en una posición de enlace entre los residentes y los facultativos docentes. Además incorpora otra serie de funciones administrativas, docentes, asistenciales, investigadoras y una función considerada "humanitaria".

Las funciones administrativas conllevan la implicación en las instituciones y la estructura docente del hospital; las funciones docentes incluyen la supervisión de los programas de formación de cada especialidad, así como la revisión de la oferta formativa complementaria del centro. El Jefe de Residentes se encarga de analizar cómo mejorar esta oferta, potenciándola y ampliándola en lo posible, todo ello bajo una estrecha relación de trabajo con tutores y jefe de estudios.

A su vez, el Jefe de Residentes mantiene una actividad asistencial que le permite permanecer vinculado a su especialidad, desarrollando su propia formación, y que le otorga una cercanía a la actividad diaria. Por último, las funciones humanitarias comprenden todas aquellas acciones relacionadas con la solución de conflictos, la atención a problemas personales o a dificultades de adaptación de los médicos residentes, así como con la mejora, en general, de la vida social de éstos en el centro docente. En conjunto, un Jefe de Residentes debe ser capaz de desarrollar tareas de planificación, organización, motivación y supervisión.

El Jefe de Residentes tiene el deber de fomentar la interrelación entre los residentes y la Comisión de Docencia. Debe ser una figura accesible y asequible para los residentes, con quien se pueda analizar los posibles problemas que surjan, discutir las soluciones más apropiadas y llevarlas a efecto, bajo el amparo y la aprobación del Jefe de Estudios y de la Comisión de Docencia. Además el Jefe de Residentes debe ser referente para atender el aspecto humano de los especialistas en formación, intentando solventar en la medida de lo posible aquellos problemas de tipo personal relacionados con el periodo de formación.

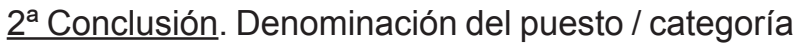
profesional:

Jefe de Residentes del hospital o del área correspondiente.

Facultativo especialista de área. Vinculación compartida con su servicio de origen y con la Docencia.

$\underline{3^{a} \text { Conclusión. Titulación exigida: }}$

Licenciatura en Medicina, Farmacia, Biología, Bioquímica, Química, o Física.

Título de Especialista obtenido el mismo año de la convocatoria, de especialidades hospitalarias para Jefe de Residentes de Hospital, o bien Título de Especialista en MFYC el mismo año de la convocatoria para Jefe de Residentes de Área.

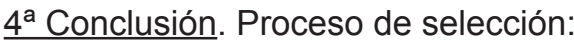

Lo realizará un tribunal o comité compuesto por: el Jefe de Estudios, los Jefes de Residentes anteriores, los Vocales de Residentes de la Comisión de Docencia, y un representante de la Dirección Médica del hospital o área correspondiente.

Se tendrán en cuenta los siguientes apartados para su evaluación:

1. Memoria: el candidato expondrá brevemente sus conocimientos acerca de las figuras docentes y presentará su propuesta de proyecto a realizar como Jefe de Residentes.

2. Currículum Vitae. 
3. Entrevista personal con el tribunal evaluador, en la que se tendrán en cuenta aptitudes, experiencia previa en el área docente, compromiso de dedicación y formación.

$\underline{5^{a} \text { Conclusión. Mecanismo del nombramiento: }}$

El Jefe de Residentes será designado por la Dirección Médica, a propuesta del Jefe de Estudios y de la Comisión de Docencia.

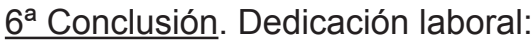

Un $40 \%$ de su jornada laboral con dedicación a actividades y funciones relacionadas con la Docencia y un $60 \%$ de actividad asistencial, lo que viene a ser en la práctica 2 jornadas semanales para tareas docentes y 3 jornadas semanales para labores asistenciales.

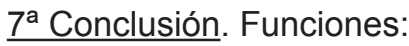

1. Colaborar con el Jefe de Estudios en la organización y coordinación de actividades docentes del Hospital. Se realizarán reuniones específicas con residentes de las diversas especialidades con el objetivo de:

- Revisar el cumplimiento de los planes de formación.

- Fomentar la realización de los programas señalados.

- Fomentar la participación en actividades docentes e investigadoras.

- Realizar informes de las reuniones que se presentarán al Jefe de Estudios y a la Comisión de Docencia.

2. Servir de enlace entre las estructuras docentes y administrativas del Hospital con los médicos residentes.

3. Organizar y supervisar las sesiones docentes organizadas por los médicos residentes.

4. Actuar como la figura de referencia frente a problemas que el colectivo de residentes y los propios residentes a nivel individual se puedan plantear, estableciendo mecanismos y procedimientos que le permitan encauzar y corregir cuantas anomalías y problemas se produzcan.

\section{$\underline{8^{\mathrm{a}} \text { Conclusión. Reconocimiento: }}$}

El Jefe de residentes es un Facultativo con funciones asistenciales y docentes, y como tal ha de ser remunerado.
Actualmente tiene el mismo reconocimiento económico y en desarrollo profesional que cualquier otro facultativo de su misma especialidad. Dicho reconocimiento económico no queda penalizado por la posibilidad que tiene el Jefe de Residentes de realizar guardias propias de su especialidad, actividad que exige gran esfuerzo a los Jefes de Residentes, debido a que su dedicación a tareas docentes y asistenciales, en los términos descritos anteriormente, no facilita el descanso posterior a la guardia.

Finalmente, en cuanto al desarrollo profesional se refiere, en el taller se consensuó que debe existir por parte del Centro/Área una disposición activa para facilitar el posterior desarrollo profesional del Jefe de Residentes en dicho centro, considerándose un valor añadido y discriminativo el haber desempeñado estas funciones en los procesos de adjudicación de plazas de Facultativo en el Centro/ Área.

\section{EL TÉCNICO DOCENTE DE LAS UNIDADES DO- CENTES}

\section{Orígenes}

Como primer antecedente podemos citar la orden ministerial de 19 de diciembre de $1983^{5}$ por la que se regula el desarrollo de la formación en atención primaria de Salud de la Especialidad de Medicina Familiar y Comunitaria, que incluye en su artículo $6^{\circ}$ las funciones del Técnico en Salud Pública y Medicina Comunitaria. Esta figura queda adscrita al Programa de MFyC, y se ubica en las Direcciones Provinciales del INSALUD.

En agosto de 1989 se aprobaron las plantillas orgánicas correspondientes a las Áreas de Salud del territorio INSALUD; en ellas figura la adscripción de un Técnico de Salud Pública de Atención Primaria a cada Área para asumir funciones relacionadas con la Salud Pública.

La orden ministerial de 22 de junio de $1995^{2}$ adscribe los Técnicos de Salud Pública a las Unidades Docentes de Medicina Familiar y Comunitaria.

El último programa formativo de la especialidad de Medicina Familiar y Comunitaria, publicado por la Orden SCO/1198/20056, define a los Técnicos en Salud Pública como aquellos profesionales que, sin ser tutores de Medicina Familiar y Comunitaria, están encargados de contribuir en la formación teórico-práctica y de investigación contemplada en 
el programa que se lleve a cabo en la Unidad Docente.

Finalmente es destacable que en los borradores previos del Real Decreto 183/2008 se incluyera la figura del Técnico de Salud o Técnico de Docencia con unas funciones similares. La versión final del $\mathrm{RD}^{3}$ deja abierta la posibilidad en su artículo 13 a que las comunidades autónomas, según sus características y criterios organizativos propios, puedan crear otras figuras docentes con la finalidad de amparar colaboraciones significativas en la formación especializada, objetivos de investigación, desarrollo de módulos genéricos o específicos de los programas o cualesquiera otras actividades docentes de interés, que son todas ellas, funciones y actividades que han sido históricamente propias de los técnicos de salud pública en atención primaria.

\section{Justificación}

La complejidad creciente de la gestión de la formación especializada a nivel de unidades docentes y centros sanitarios con acreditación docente hace necesaria la profesionalización de la misma mediante la incorporación de figuras de apoyo que según su complejidad se incorporen a la estructura tradicional de docencia (comisiones de docencia, jefes de estudios, tutores y administrativos).

Los técnicos en salud pública de atención primaria poseen en la actualidad una elevada heterogeneidad en sus funciones, incluso entre áreas de salud de una misma comunidad autónoma, llegando en algunos casos a no realizar funciones de apoyo a la gestión de la docencia. Asimismo, algunos centros sanitarios docentes han incorporado a partir del RD $183 / 2008^{3}$ técnicos docentes que, a falta de legislación autonómica, necesitan de criterios comunes a sus homónimos de atención primaria que guíen sus actuaciones.

Los técnicos de salud pública de atención primaria son un colectivo profesional cuyo reconocimiento profesional es bajo, dado que su reconocimiento salarial es inferior al de categorías profesionales equivalentes, generalmente no tienen acceso a complementos a los que sí acceden estas categorías equivalentes, e incluso no se les reconoce la prestación de servicios a tiempo completo como méritos en las convocatorias de procesos selectivos a puestos de categorías profesionales a los que, paradójicamente, contribuyen a formar. Al ser una categoría profesional joven posee en general elevados niveles de interinidad además de escasas convocatorias de oposiciones para consolidación de plazas.

Esto hace del RD 183/2008 y su posterior desarrollo una herramienta para mejorar la estructura y el funcionamiento de unidades docentes y centros sanitarios con acreditación docente. La derogación de la legislación anterior, que hacía referencia a los técnicos de salud de atención primaria y la cesión a las comunidades autónomas a través del mencionado RD de la potestad de regular y adaptar en su ámbito de aplicación otras figuras docentes, supone una oportunidad de mejora para el reconocimiento profesional y la situación laboral de los técnicos docentes que desarrollan su trabajo tanto en atención primaria como en los centros hospitalarios acreditados como docentes.

Partiendo de esta figura previa, entendemos que esta argumentación debe servir para solicitar a las comunidades autónomas que recojan la figura del Técnico Docente en sus normativas docentes, en la mayoría de casos aún por desarrollar, incluyendo también las medidas laborales y profesionales necesarias para garantizar el correcto desempeño de estos profesionales clave en el progreso y crecimiento de las estructuras de gestión de la formación sanitaria especializada.

\section{Conclusiones y propuestas}

- Definición de la figura del técnico docente.

El Técnico Docente es el profesional con formación acreditada en metodología docente e investigadora que, dentro de la estructura de gestión docente y sin ser tutor de residentes, lleva a cabo las tareas encomendadas por la Comisión de Docencia y el Jefe de Estudios de formación especializada, encaminadas a la ejecución de los programas teórico-prácticos de las especialidades en formación sanitaria especializada acreditadas en el centro sanitario y/o unidad docente, desempeñando funciones de apoyo a la calidad e investigación en la docencia, y participando en las actividades que se desarrollen en este ámbito, dirigidas a la mejora de la formación tanto de los residentes como la de los propios tutores y el resto de figuras docentes, y profesionales en general del ámbito sanitario.

\section{$\underline{1^{\mathrm{a}} \text { Conclusión. }}$}

Se acuerda que la denominación del Técnico Docente ha de completarse especificando la dedicación dentro del ámbito sanitario, con el objetivo de evitar equívocos con los Técnicos Docentes de otras profesiones. En base al RD 183/2008 en el 
cuál se definen otras figuras docentes en la Formación Sanitaria Especializada (FSE), se propone la denominación: Técnico Docente de Formación Sanitaria Especializada.

Pero teniendo en cuenta las tareas que actualmente desempeñan los Técnicos Docentes (Formación Sanitaria Especializada y Formación Continuada) se propone la denominación: Técnico Docente Sanitario.

En caso de optar por la primera denominación propuesta, sería necesaria la creación de otra Figura Docente que se ocupase de la Formación Continuada, y que debería denominarse como Técnico Docente de Formación Continuada Sanitaria.

- Denominación del puesto/categoría profesional.

Técnico Docente Sanitario / Técnico Docente de Formación Sanitaria Especializada.

\section{$\underline{2^{\mathrm{a}} \text { Conclusión }}$}

En cuanto a la categoría profesional, se acepta por unanimidad que ha de tener categoría de gestión asistencial, igual a la que tenía con anterioridad al nombramiento.

- Titulación exigida.

Título de Especialista en Ciencias de la Salud, que se corresponda con alguna de las especialidades acreditadas en el Centro Sanitario y/o Unidad Docente.

\section{$\underline{3^{a} \text { Conclusión }}$}

Se acepta la posibilidad de acceso a esta categoría o figura docente de personal procedente de otras titulaciones como Pedagogía, siempre y cuándo exista previamente un Técnico Docente con especialidad sanitaria correspondiente a su Unidad Docente contratado en dicha Unidad.

- Mecanismo del nombramiento.

El número de Técnicos Docentes dependerá de las peculiaridades de cada centro sanitario o unidad docente: número de residentes en formación, centros docentes adscritos, dispersión geográfica, otras actividades, etc.

El nombramiento del técnico se debe efectuar a través de un concurso de méritos de carácter abierto y competitivo, bajo los principios constitucionales de igualdad, mérito y capacidad, con sujeción a los criterios generales que en su caso apruebe la Comisión de Recursos Humanos del Sistema Nacional de Salud, entre profesionales que presten servicios en los distintos dispositivos integrados en el centro sanitario o unidad docente, que ostenten el título de especialista requerido, y que acrediten formación en metodología docente e investigadora.

El técnico docente será nombrado por el órgano directivo del centro a propuesta de la comisión de docencia, oídos los propios profesionales por un periodo de 1-4 años, renovable, previa ratificación por la comisión de docencia.

\section{4a Conclusión.}

Tras el análisis de las funciones del Técnico Docente y formación específica docente requerida para el desarrollo del puesto, se llego al consenso de que la duración de estos contratos debía ser mínimo de un año y máximo de cuatro.

Se aprueba por unanimidad que dicho contrato sea renovable, y que dicha renovación esté justificada por la memoria de la actividad realizada y por el proyecto presentado para el próximo periodo. Dicho proyecto ha de tener explicitada la dedicación a la Unidad Docente.

También se propone un sistema de renovación del cargo escalonado con el sistema de renovación del Jefe de Estudios para dar continuidad a la Unidad Docente.

- Funciones.

Las funciones que se definen en este documento de consenso son las que actualmente desarrollan los Técnicos Docentes, pero quedan pendientes de cómo se defina dicha figura en los correspondientes Decretos Autonómicos (ver punto de consenso $\left.n^{\circ} 1\right)$.

- Formar parte de la Comisión de Docencia.

- Formar parte de la Comisión de Formación Continuada.

- Colaborar con el Jefe de Estudios y la Comisión de Docencia en la planificación y acreditación de las actividades formativas transversales que se programen por el centro sanitario y/o la comunidad autónoma, así como en su adaptación a las características propias del correspondiente centro/ unidad docente.

- Impartir los cursos y módulos que contemplan tanto los programas transversales de 
formación como los específicos que contienen los programas de formación sanitaria especializada acreditados en el centro.

- Colaborar con el jefe de estudios del centro/unidad docente en la coordinación de las actividades de formación especializada con las restantes actividades formativas (grado, formación continuada, programa de visitantes,...) que se lleven a cabo en el mismo centro/unidad.

- Asesorar a las unidades docentes acreditadas y apoyar a los tutores de las mismas, detectando sus necesidades docentes y proporcionando oportunidades para facilitar su formación.

- Monitorización y apoyo técnico en los centros sanitarios acreditados para la docencia, detectando y analizando las deficiencias formativas de los mismos.

- Elaboración y mantenimiento de los programas de gestión de la calidad en el correspondiente centro/unidad docente, así como el desarrollo de las actividades de mejora de la calidad definidas en dicho plan.

- Apoyo técnico en la preparación de las auditorías del Plan Nacional de Auditorias docentes y en la elaboración y seguimiento de los planes de mejora resultantes de las mismas.

- Estimular, apoyar y crear líneas sólidas de investigación en el centro/unidad docente, enmarcándolas en las necesidades de cada una de ellas y en los planes generales de la comunidad autónoma.

- Colaborar en la coordinación de las actividades formativas que desarrollan otros centros/unidades docentes (medicina de familia, medicina preventiva y salud pública, unidades de salud mental, especialidades de enfermería,...) que incluyen al centro del técnico docente dentro su propia acreditación.

- Colaboración con la Formación de Pregrado, coordinando rotaciones de los estudiantes de Ciencias de la Salud.

- Colaboración con la Formación continuada, desarrollando y/o impartiendo cursos de formación continuada para los profesionales sanitarios de su área correspondiente.

\section{- Dedicación laboral.} $\underline{5^{a} \text { Conclusión. }}$

En cuanto al apartado de las funciones y dedicación, se aceptó la posibilidad de una mínima de- dicación asistencial a su especialidad sanitaria de origen, con el fin de evitar que el profesional que temporalmente dedica su actividad profesional a tareas de gestión de la docencia sea penalizado en su carrera profesional y/o en su salario, con respecto a sus compañeros de especialidad.

Dicha propuesta de dedicación docente y asistencial compartida ha de quedar implícita en el proyecto para acceder/renovar el cargo, y ha de estar aprobada por la Dirección-Gerencia. Se propone que la dedicación asistencial pudiera ser del 10$20 \%$, equivalente a 1 jornada cada 15 días - 1 jornada cada semana.

De este modo, en los criterios para acreditación - reacreditación podrían incluir aspectos tanto docentes y de gestión, como asistenciales y de investigación.

- Acreditación - reacreditación.

Los técnicos docentes estarán sujetos a una serie de requisitos de acreditación - reacreditación, debiéndose regular los procedimientos de evaluación periódica del desempeño de sus labores.

Según el RD 183/2008, a efectos de acreditación y reacreditación, se tendrán en cuenta, entre otros factores, la experiencia profesional continuada como especialista, la experiencia docente, las actividades de formación continuada, la actividad investigadora y de mejora de calidad, la formación específica en metodología docente, así como el resultado de las evaluaciones de calidad y encuestas sobre el grado de satisfacción alcanzado.

- Reconocimiento salarial.

Equiparación del reconocimiento salarial neto del técnico docente a la de la categoría profesional equivalente (FEA de la especialidad correspondiente, Médico de Familia en Equipos de Atención Primaria, FEA de Medicina Preventiva y Salud Pública, Enfermería Especialista, etc.).

En todos los casos, cuando el Técnico Docente lleve a cabo actividades complementarias o actividades fuera del horario laboral, debe contemplarse la posibilidad de abonar el correspondiente complemento por actividades complementarias y por Jornada complementaria. 


\section{AGRADECIMIENTOS}

Ponentes en la Sesión del Jefe de Residentes: Antonio Ríos Zambudio, Raquel Gómez Bravo, Álvaro de Casa Fernández e Irene Villegas Martínez. Ponentes Técnico Docente: José Saura Llamas, José Jonay Ojeda Feo, Carmen Botella Martínez, Francisco Sánchez Marín. Y a todos los participantes por sus valiosas aportaciones.

\section{BIBLIOGRAFÍA}

1. Real Decreto $127 / 1984$, de 11 de enero, por el que se regula la Formación Médica Especializada y la obtención del Título de Médico Especialista. BOE 11 de Enero 1984.
2. Orden de 22 de junio de 1995 , por la que se regulan las Comisiones de Docencia y los sistemas de evaluación de la formación de los médicos y farmacéuticos especialistas. Ministerio de la Presidencia. BOE 30 de junio 1995.

3. Real Decreto $183 / 2008$, de 8 de febrero, por el que se determinan y clasifican las especialidades en Ciencias de la Salud y se desarrollan determinados aspectos del sistema de formación sanitaria especializada. Ministerio de la Presidencia. BOE 21 de Febrero 2008.

4. Ley de ordenación de las profesiones sanitarias. BOE 21 de noviembre 2003.

5. Orden de 19 de diciembre de 1983, por la que se regula el desarrollo de la formación en atención primaria de Salud de la Especialidad de Medicina Familiar y Comunitaria. BOE 22 de diciembre 1983.

6. Orden SCO/1198/2005, de 3 de marzo, por la que se aprueba y publica el programa formativo de la especialidad de Medicina Familiar y Comunitaria. BOE 3 de mayo de 2005. 